\section{Optic disc parameters in manifest and suspected glaucoma}

\author{
Peter Wanger, ${ }^{1}$ Lucian Vancea, ${ }^{2}$ \\ Lene Martin ${ }^{1,3}$
}

'Department of Clinical Neuroscience, Ophthalmology \& Vision, Karolinska Institutet, Stockholm; ' ${ }^{2}$ ye Clinic, Sundsvalls hospital, Sundsvall; ${ }^{3}$ Academy of Health and Welfare, Mälardalen University, Eskilstuna, Sweden

\section{Abstract}

Structure and function measurements are important in glaucoma management. Digital fundus photography has become a standard procedure and the Heidelberg Retina Tomograph (HRT), commonly used by glaucoma specialists, provides a glaucoma probability score (GPS). The visual field index (VFI) is a novel statistic, aiming to facilitate follow-up of glaucoma patients. The aim of this study was to compare the results from the digital analysis of fundus photographs with HRT measurements including GPS and VFI in patients with ocular hypertension, suspect glaucoma or glaucoma, and if possible define an optic disc index, useful in glaucoma diagnosis. Fifty-eight consecutive patients from a glaucoma service were included. Optic disc parameters (disc and cup areas) were measured on digital fundus photographs, using a semi-automatic method, and compared with the GPS from the HRT and the VFI from standard automated perimetry. A significant relationship was observed between the GPS group classification (normal, borderline, or abnormal) and VFI classification (normal or abnormal), both when the GPS borderline group was regarded as normal $(\mathrm{P}=0.0038$ Fisher test $)$ and as abnormal $(\mathrm{P}=0.0179$, kappa $=0.33)$. No significant relationship was observed between VFI and optic disc parameters. The threedimensional information in the GPS appears to be more related to visual function, as measured by VFI, than the planimetric measures of the optic disc.

\section{Introduction}

Digital fundus cameras are a standard part of the ophthalmologic equipment, commonly used in screening for retinal disorders, such as age-related macular degeneration. ${ }^{1}$ Evaluation of the optic disc parameters on fundus photographs is clinically relevant in glaucoma diag- nosis and follow-up ${ }^{2}$ and several algorithms have been evaluated for planimetric calculation of optic nerve head parameters. ${ }^{3}$ Optic disc analysis can also be automatically performed with confocal scanning laser ophthalmoscopy ${ }^{4}$ using the Heidelberg Retinal Tomography, HRT (Heidelberg Engineering $\mathrm{GmbH}$, Heidelberg, Germany).

Recently, we developed a program which was designed to provide clinically relevant measures of optic disc parameters with a minimum of user input. ${ }^{5}$ The program can be used directly on the acquired images, with the examined subject still available for re-examination.

In topographic analyses of the optic nerve the effect of disc size has to be taken into account. ${ }^{6}$ When calculating linear or area cupto-disc ratios, small discs may be classified normal despite the presence of glaucoma and, vice versa, large discs may be falsely labelled as glaucomatous. ${ }^{7}$ Recently, a scoring system for evaluation of the optic disc in glaucoma was presented by the RAND study group ${ }^{8}$, which relied on estimation of both the cup-todisc ratio and the disc size in order to overcome this drawback.

The aim of this study was to compare the results from the digital analysis of fundus photographs with HRT measurements including glaucoma probability score (GPS) and visual field index (VFI) in patients with ocular hypertension, suspect glaucoma, or glaucoma, and, if possible, define an optic disc index, useful in glaucoma diagnosis.

\section{Materials and Methods}

\section{Subjects}

The patients were recruited from a glaucoma service at a regional hospital. Inclusion criteria were as follows: primary open angle glaucoma (POAG), defined as intraocular pressure (IOP) $>21 \mathrm{mmHg}$ at two or more occasions AND either retinal nerve fiber layer (RNFL) defect or visual field (VF) defect, without other explanation, or both; suspected glaucoma, defined as either IOP $>21 \mathrm{mmHg}$ at two or more occasions OR either retinal RNFL defect or VF defect, without other explanation; ocular hypertension (OHT), defined as IOP >21 $\mathrm{mmHg}$ at two or more occasions with normal VF and no RNFL defect; and, normal tension glaucoma (NTG), defined as IOP $<22 \mathrm{mmHg}$ at two or more occasions AND either RNFL defect or VF defect, without other explanation, or both. Exclusion criteria were other disorders (such as optic nerve hypoplasia) that could influence the eye or vision and subjects with unreliable visual field results, defined according to the manufacturer's manual.

All patients had been examined at least
Correspondence: Lene Martin, Academy of Health and Welfare, Mälardalen University, P0 Box 325, SE-631 05 Eskilstuna, Sweden.

Tel. +46.161.532.02 - E-mail: lene.martin@mdh.se

Key words: digital fundus photography, optic disc, heidelberg retina tomograph, glaucoma probability score, visual field index.

Received for publication: 13 April 2011. Accepted for publication: 18 October 2011.

This work is licensed under a Creative Commons Attribution NonCommercial 3.0 License (CC BYNC 3.0).

(C) Copyright P. Wanger et al., 2011

Licensee PAGEPress, Italy

Eye Reports 2011; 1:e12

doi:10.4081/eye.2011.e12

twice at the glaucoma service and were scheduled for follow-up. One eye from each of the 58 patients was randomly selected for analysis (Tables 1 and 2). All but 16 of the subjects had refraction within $\pm 3 \mathrm{D}$ (all refraction is expressed in spherical equivalent). One subject had a hyperopia of $+3.75 \mathrm{D}$. The remaining subjects had moderate myopia $(-3.5 \mathrm{D}$ to $-6 \mathrm{D}$; $\mathrm{n}=7$ ) or high myopia (-6.75D to $-14.75 \mathrm{D} ; \mathrm{n}=8)$.

\section{Methods}

All subjects underwent a standard clinical examination and visual field examination, using the Humphrey Visual Field Analyser (HFA), 24-2 Threshold test, Sita Fast (Carl Zeiss Meditec, Dublin, California, USA). Digital images of the optic nerve head $(\mathrm{ONH})$ were obtained using the Heidelberg Retinal Tomography 3 (HRT3) (Heidelberg Engineering $\mathrm{GmbH}$ ) and the Zeiss VISUPAC and FF 450plus telecentric fundus camera system (Carl Zeiss Meditec). Measurements of optic disc parameters were performed using the GPS calculations in the HRT. GPS makes a global assessment of the ONH by comparing the measured shape of the optic disc and the surrounding retina to a model for normal and glaucomatous optic nerves. The output of the program is a number between 0.00 and 1.00 , which is classified as within normal limits (below 0.28), borderline ( 0.28 to 0.64 ) or outside normal limits (above 0.64). ${ }^{9}$ The quality of the images is evaluated by the HRT software and classified as excellent, very good, good, acceptable, poor or not usable (manufacturer's manual). Only recordings with quality grade acceptable or better were used. The global GPS measure and the cup area (CA) measure were used.

The Retinal Size Tool (RST) was used for measurements on the digital fundus photographs. ${ }^{5}$ RST is a computer program developed in-house, that can be used on any digital fun- 
dus photograph where both the macula and the $\mathrm{ONH}$ are visible. Optic disc parameters, i.e. optic disc and cup, are assumed to be elliptical, ${ }^{10}$ and the user marks the endpoints of their long and short diameters by mouse clicks. In order to compensate for differences in magnification due to camera and eye optics, the macula-optic disc centre distance is used as reference measure ${ }^{10-12}$ when converting pixel units to metric distance. ${ }^{5}$ This measure is reported to be quite constant among adults. ${ }^{13}$ The interoperator agreement has been shown to be very good, both regarding disc and cup area $(\mathrm{r}=0.92$ and 0.93 , respectively). ${ }^{5}$ In the current study the cup area (CA), disc area (DA), and the quotient cup/disc area (CADA) were evaluated.

The $\mathrm{VFI}^{14}$ in the HFA was used for comparison with the structural measures. An HFA VFI $>98 \%$ was regarded as a normal (Bengtsson, personal communication).

The study was performed according to the Helsinki declaration and approved by the local ethical committee. Written informed consent was obtained from all patients prior to enrolment.

\section{Statistics}

For comparisons and correlations, the Fisher test, the Kruskall-Wallis non-parametric ANOVA-test and the Spearman Correlation test were used. Binary logistic regression was used in an attempt to define a diagnostic index based on the measurement on the fundus photographs.

\section{Results}

\section{Optic disc parameters in Heidelberg Retina Tomograph 3 and Retinal Size Tool}

Table 3 show the disc parameters in all subjects measured both with the RST (CA, DA, CADA) and the HRT GPS (CA). There was a strong correlation in the total group $\left(\mathrm{r}^{2}=0.63\right.$; $\mathrm{P}<0.0001)$ between the CA measurements performed by the GPS and the RST, and no difference in median value ( 0.46 in both measures). The CA's were significantly smaller in the high myopes, measured both with RST (median 0.33; $\mathrm{P}<0.0001$ ) and GPS (median 0.32; $\mathrm{P}=0.004$ ).

Nineteen patients out of 58 (33\%) had normal optic discs according to the GPS program; 12 were judged as borderline, and 27 as abnormal (example discs shown in Figure 1). In the 8 patients with high myopia, 7 were classified as normal and 1 as borderline. Table 4 shows the disc parameters, measured by RST, in the three GPS groups. A significant difference was found in CA and CADA between the normal group and both the borderline and the abnormal group and in DA between the normal and the abnormal group (Table 5). CADA correlated significantly, but weakly with GPS $\left(\mathrm{r}^{2}=0.19, \mathrm{P}<0.001\right)$.

Optic disc parameters and humphrey visual field analyzer visual field index

Eighteen patients out of 58 (31\%) had nor- mal visual fields according to the VFI, and 40 had subnormal VFI values. In the 8 patients with high myopia, 5 had normal VFI values. Table 6 shows the relationship between HFA VFI and disc parameters in all patients. There

Table 1. Age, gender and refraction (spherical equivalent).

\begin{tabular}{ll}
\hline Median age (range) & $64(30-85)$ \\
Gender (female/male) & $31 / 27$ \\
\hline Median refraction (range) & $-0.63(-14.5$ to +3.75$)$ \\
\hline
\end{tabular}

Refraction expressed as spherical equivalent.

Table 2. Diagnoses in the studied group.

$\begin{array}{cccccccc} & \text { OHI POAG } & \text { NIG } & \begin{array}{c}\text { Glaucoma } \\ \text { secondary to } \\ \text { pseudoexfoliation }\end{array} & \begin{array}{c}\text { Glaucoma } \\ \text { secondary to } \\ \text { pigmentary } \\ \text { dispersion }\end{array} & \begin{array}{c}\text { Suspect } \\ \text { glaucoma }\end{array} \\ \text { Number of subjects } & 7 & 13 & 10 & 15 & 3 & 10\end{array}$

Suspected glaucoma consisted of POAG, NTG, and pseudoexfoliative glaucoma.

Table 3. Median values for disc parameters in relation to the degree of myopia.

\begin{tabular}{lccc} 
& All $(\mathrm{n}=58)$ & High myopes $(\mathrm{n}=8)$ & Non high myopes $(\mathrm{n}=50)$ \\
Median RST DA $\left(\mathrm{mm}^{2}\right)$ & $2.17(1.19-3.23)$ & $1.64(1.42-2.14)$ & $2.22(1.19-3.23)$ \\
Median RST CA $\left(\mathrm{mm}^{2}\right)$ & $0.46(0.12-1.89)$ & $0.33(0.12-0.41)$ & $0.56(0.12-1.89)$ \\
\hline Median RST CADA $\left(\mathrm{mm}^{2}\right)$ & $0.11(0.04-0.20)$ & $0.1(0.05-0.19)$ & $0.11(0.04-0.20)$ \\
Median HRT GPS CA $\left(\mathrm{mm}^{2}\right)$ & $0.46(0.09-1.11)$ & $0.32(0.15-0.30)$ & $0.48(0.09-1.11)$ \\
\hline
\end{tabular}

RST, retinal size tool; DA, disc area; CA, cup area; CADA, cup/disc area quotient; HRT, Heidelberg retinal tomography; GPS, glaucoma probability score. Values in parentheses represent the range of values.

Table 4. Median values for retinal size tool disc parameters and humphrey visual field analyzer visual field index in the three glaucoma probability score classification groups.

GPS normal GPS borderline GPS abnormal

\begin{tabular}{lccc} 
Number of subjects & 19 & 12 & 27 \\
Median RST DA $\left(\mathrm{mm}^{2}\right)$ & $1.67(1.19-3.02)$ & $2.15(1.31-2.68)$ & $2.32(1.38-3.23)$ \\
\hline Median RST CA $\left(\mathrm{mm}^{2}\right)$ & $0.31(0.12-0.65)$ & $0.59(0.13-1.04)$ & $0.63(0.17-1.89)$ \\
Median RST CADA $\left(\mathrm{mm}^{2}\right)$ & $0.16(0.07-0.32)$ & $0.28(0.1-0.41)$ & $0.28(0.08-0.59)$ \\
\hline Median HFA VFI $(\%)$ & $99(65-100)$ & $97.5(80-100)$ & $97(65-99)$ \\
\hline
\end{tabular}

RST, retinal size tool; GPS, glaucoma probability score; DA, disc area; CA, cup area; CADA, cup/disc area quotient; HFA, humphrey visual field analyzer; $\mathrm{VFI}$, visual field index. Values in parentheses represent the range of values.

Table 5. ANOVA P-values of comparisons between GPS classifications for cup area, disc area, and cup/disc area quotient.

\begin{tabular}{lccc} 
GPS classification & CA & DA & CADA \\
Normal $v$ borderline & $<0.05$ & $>0.05$ & $<0.05$ \\
\hline Normal $v$ s abnormal & $<0.001$ & $<0.001$ & $<0.01$ \\
Borderline s abnormal & $>0.05$ & $>0.05$ & $>0.05$ \\
\hline
\end{tabular}

CA, cup area; DA, disc area; CADA, cup/disc area quotient; GPS, glaucoma probability score; VFI, visual field index.

Table 6. Relationship between humphrey visual field analyzer visual field index and median disc parameters.

VFI normal (>98\%) VFI abnormal $(<=98 \%)$

\begin{tabular}{lcc} 
Number of subjects & 18 & 40 \\
Median DA $\left(\mathrm{mm}^{2}\right)$ & $2.01(1.19-3.02)$ & $2.21(1.23-3.23)$ \\
\hline Median CA $\left(\mathrm{mm}^{2}\right)$ & $0.43(0.12-0.96)$ & $0.5(0.12-1.89)$ \\
Median CADA $\left(\mathrm{mm}^{2}\right)$ & $0.22(0.07-0.4)$ & $0.23(0.07-0.59)$ \\
\hline Median HRT GPS CA $\left(\mathrm{mm}^{2}\right)$ & $0.17(0.02-0.7)$ & $0.64(0.08-0.92)^{*}$
\end{tabular}

VFI, visual field index; DA, disc area; CA, cup area; CADA, cup/disc area quotient; HRT, Heidelberg retinal tomography; GPS, glaucoma probability score; ${ }^{*} \mathrm{P}=0.001$. Values in parentheses represent the range of values. 
Table 7. Relationship between glaucoma probability score (GPS) grouping and normal/ abnormal visual field index, when the GPS borderline group was regarded as normal.

\begin{tabular}{lcc} 
& $\begin{array}{c}\text { GPS normal or } \\
\text { borderline }\end{array}$ & GPS abnormal \\
$\mathrm{VFI}>98$ & 15 & 3 \\
$\mathrm{VFI} \leq=98$ & 16 & 24 \\
\hline
\end{tabular}

GPS, glaucoma probability score; VFI, visual field index; P, 0.0038 Fisher test; kappa $=0.36$.

Table 8. Relationship between glaucoma probability score (GPS) grouping and normal/ abnormal visual field index, when the GPS borderline group was regarded as abnormal.

\section{GPS normal GPS borderline} or abnormal

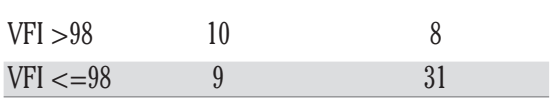

GPS, glaucoma probability score; $\mathrm{VFI}$, visual field index; $\mathrm{P}=0.0179$ Fischer test; kappa $=0.33$.
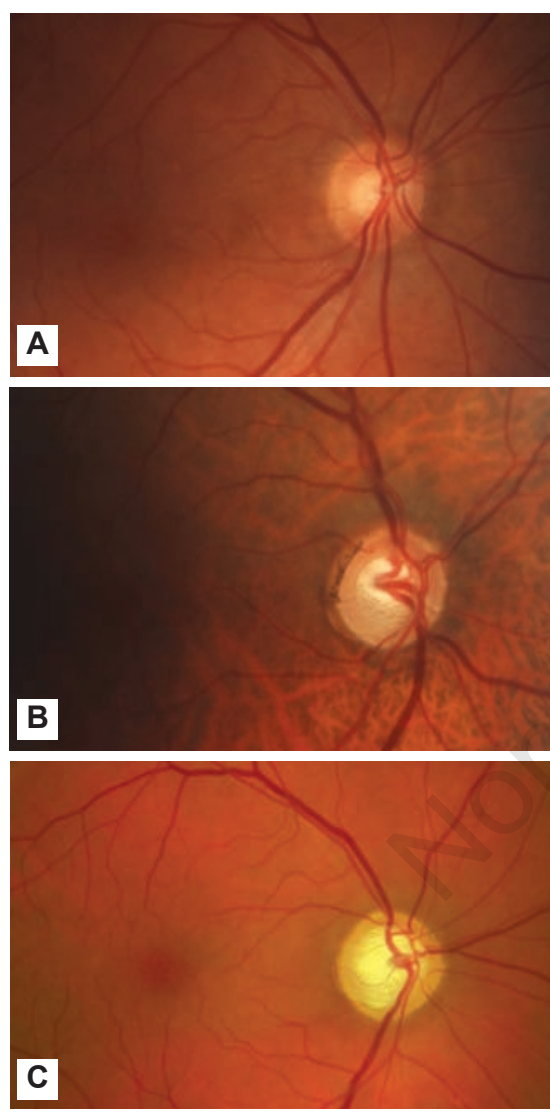

Figure 1. Examples of optic discs with different glaucoma probability score classifications and visual field index findings. (A) Female born 1944, with OHT, GPS normal, RST CA/DA 0.19,VFI 99\%; (B) Male born 1938, with pseudoexfoliation, GPS borderline, RST CA/DA 0.22, VFI 93\%; and (C) Male born 1948, with NTG, GPS abnormal, RST CA/DA 0.64, VFI 83\%.OHT, ocular hypertension; GPS, glaucoma probability score; RST, Retinal Size Tool; CADA, cup/disc area quotient in $\mathbf{m m}^{2}$; VFI, visual field index; NTG, normal tension glaucoma. was a significant difference between VFI in the GPS normal group compared with the GPS abnormal group $(<0.05)$ (Table 7). The correlation between VFI and GPS score was weak $\left(r^{2}=0.083, P=0.027\right)$. Regarding classification, a significant relationship was observed between the GPS group classification (normal, borderline, or abnormal) and VFI classification (normal or abnormal), both when the GPS borderline group was regarded as normal and as abnormal (Tables 7 and 8). There was no correlation between CADA and VFI $\left(\mathrm{r}^{2}=0.006, \mathrm{P}=\right.$ 0.56). A logistic regression equation based on DA and CADA did not discriminate between subjects with normal and abnormal VFI.

\section{Discussion}

\section{Optic disc parameters in}

\section{Heidelberg Retina Tomograph 3} and Retinal Size Tool and GPS

\section{classifications}

In a previous study, comparing disc measurements from RST and HRT3 in non-glaucomatous subjects, no significant difference between the measurements were found either in DA or in CA. ${ }^{5}$ This observation was confirmed regarding CA in the current study of glaucoma subjects, but could not be confirmed regarding the DA, since the GPS program does not report this measure.

It is well known that disc size influences the ability to classify the optic disc as normal or glaucomatous. ${ }^{6,89}$ This influence is true also for the HRT3 GPS classification. ${ }^{9}$ In the current study, the largest discs, median disc area of $2.32 \mathrm{~mm}^{2}$, were found in the GPS abnormal group, compared to median disc area of 2.15 $\mathrm{mm}^{2}$ in the GPS borderline group, and median disc area of $1.67 \mathrm{~mm}^{2}$ in the GPS normal group.

For use in glaucoma management, the RAND system ${ }^{8}$ defines a score for the linear cup to disc ratio (CDR): score of 0 is CDR < 0.5 , score 1 is CDR 0.5 to $<0.7$, score 2 is CDR 0.7 to 0.9 , and score 4 is CDR $>0.9$, and the RAND system adjusts this score by subtracting one unit if the DA is ophthalmoscopically judged to be large and adding one unit if the DA is judged to be small. In the current study, which included patients with no or low degree of glaucomatous ON damage, binary logistic regression showed no significant effects of combining optic disc parameters for the identification of eyes with abnormal VFI. Thus, the significant relationship between GPS and VFI appears to depend on the three-dimensional analysis of the neuro-retinal rim area performed by the HRT. A weakness in the RST method is that notching of the rim area is not visible in the measurement values. However, visual evaluation of the fundus photographs revealed notching in one eye only. The concordance between GPS and VFI in classification as normal and abnormal was moderate. Alencar, et al. ${ }^{15}$ reported that GPS values were predictive of conversion in a population of patients with suspected glaucoma. Thus, the combination of GPS and VFI data may provide an easily analyzed basis for decision-making in glaucoma management.

\section{Conclusions}

A statistically significant relationship was found between HRT GPS and HFA VFI, but not between optic disc parameters, obtained by planimetric measurements on digital fundus photographs. The three-dimensional information in the GPS appears to be more related to visual function, as measured by VFI, than the planimetric measures of the optic disc.

\section{References}

1. Sheidow PT, Hooper P. Prospective evaluation of digital non-stereo color fundus photography as a screening tool in age-related macular degeneration. Am J Ophthalmol 2005;139:455-61.

2. O'Leary N, Crabb DP, Mansberger SL, et al. Glaucomatous progression in series of stereoscopic photographs and Heidelberg retina tomograph images. Arch Ophthalmol 2010;128:560-8.

3. Laemmer R, Schroeder S, Martus P, et al. Quantification of neuroretinal rim loss using digital planimetry in long-term follow-up of normals and patients with ocular hypertension. J Glaucoma 2007;16:430-6.

4. Chauhan BC, Hutchison DM, Artes PH, et al. Optic disc progression in glaucoma: comparison of confocal scanning laser tomography to optic disc photographs in a prospective study. Invest Ophthalmol Vis Sci 2009;50:1682-91.

5. Bartling H, Wanger P, Martin L. Measurement of optic disc parameters on digital fundus photographs - algorithm development and evaluation. Acta Ophthalmol Scand 2008;86:837-41.

6. Heijl A, Mölder H. Optic disc diameter influences the ability to detect glaucomatous disc damage. Acta Ophthalmol (Copenh) 1993;71:122-9.

7. Hoesl LM, Mardin CY, Horn FK, et al. Influence of glaucomatous damage and optic disc size on glaucoma detection by scanning laser tomography. J Glaucoma 2009;18:385-9.

8. RAND Study Group. For which glaucoma suspects is it appropriate to initiate treat- 
ment? Ophthalmology 2009;116:710-16.

9. Strouthidis NG, Garway-Heath DF. New developments in Heidelberg retina tomograph for glaucoma. Curr Opin Ophthalmol 2008;19:141-8.

10. Williams TD. Elliptical features of the human optic nerve head. Am J Optom Physiol 0pt 1987;64:172-8.

11. Wakakura M, Alvarez E. A simple clinical method of assessing patients with optic nerve hypoplasia. The disc-macula distance to disc diameter ratio (DM/DD). Acta Ophthalmol (Copenh) 1987;65:612-7.

12. Williams TD, Wilkingson JM. Position of the fovea centralis with respect to the optic nerve head. Optom Vis Sci 1992;69;369-77.

13. Mok KH, Lee VW. Disk-to-macula distance to disc-diameter ratio for optic disc size estimation. J Glaucoma 2002;11:392-5.

14. Bengtsson B, Heijl A. A visual field index for calculation of glaucoma rate of progression. Am J Ophthalmol 2008;145:343-53.

15. Alencar LM, Bowd C, Weinreb RN, et al. Comparison of HRT-3 glaucoma probability score and subjective stereophotograph assessment for prediction of progression 